\title{
The outcomes of the postulated interaction between SARS-CoV-2 and the renin-angiotensin system on the clinician's attitudes toward hypertension treatment
}

\author{
Cenk Sarı $\mathbb{1}^{1} \cdot$ Ersin Çağrı Şimşek ${ }^{2} \cdot$ Öner Özdoğan ${ }^{1}$
}

Received: 29 May 2020 / Revised: 28 September 2020 / Accepted: 20 October 2020 / Published online: 9 November 2020

(c) The Author(s), under exclusive licence to Springer Nature Limited 2020

\begin{abstract}
Concern has arisen about the role played in coronavirus disease 2019 (COVID-19) infection by angiotensin-converting enzyme (ACE) inhibitors and angiotensin II receptor blockers (ARBs). This study was designed to assess the practice behaviors of physicians toward hypertension treatment with ACE-i or ARBs during the COVID-19 pandemic. A selfadministered survey questionnaire consisting of 26 questions about current hypertension treatment with ACE-i/ ARBs was applied to cardiologists, internists, and family physicians in central and western Turkey, between 01 and 19 May 2020. A total of 460 physicians were approached, and $220(47.8 \%)$ participated in the study. Of the total respondents, $78.7 \%$ reported that they had not changed their antihypertensive medication prescribing pattern, $8.6 \%$ of clinicians had changed ACE-i/ ARBs medicine of patients during the COVID-19 pandemic and 12.7\% of them were undecided. The median ( \pm interquartile range) score indicating general reliance level of physicians in ACE-i/ARBs therapy was $8 \pm 4$ (range, 1-10). In multiple comparison analyses, the general reliance level in ACE-i/ARBs, reliance level when starting a new ACEi/ARBs and changing behavior in heart failure patients were significantly different with regard to the specialties (p:0.02, p:0.009, p:0.005 respectively). Although most of the physicians found the publications about ACE-i/ ARBs during the COVID-19 pandemic untrustworthy, there were variable levels of knowledge and reliance among different physicians and specialty groups. In general, the ACE-i/ ARBs prescribing habits were not affected by safety concerns during the COVID-19 pandemic in Turkey.
\end{abstract}

\section{Introduction}

In the first half of 2020, it was speculated that angiotensinconverting enzyme inhibitors (ACE-i)/angiotensin receptor blockers (ARBs) may make patients more susceptible to COVID-19 disease and lead to worse outcomes. This early hypothesis led to concerns among patients taking these medications and among physicians prescribing them for long periods. Angiotensin-converting enzyme 2 (ACE2) is a crucial component of the renin-angiotensin-aldosterone system (RAAS), which metabolizes a potent vasoconstrictor

Cenk Sarı

drcenksari@gmail.com

1 Department of Cardiology, Tepecik Education and Research Hospital, University of Health Sciences Turkey, İzmir, Turkey

2 Department of Cardiology, Balıkesir State Hospital, Balıkesir, Turkey angiotensin II to vasodilator peptide angiotensin [1]. This hypothesis was based on the fact that coronavirus 2 (SARSCoV-2) that causes COVID-19 disease gains entry into cells by binding to the ACE2 receptor [2-4]. Moreover, some previous studies have suggested that ACE-i/ARBs blocker treatment up-regulates ACE expression and the use of these drugs may increase susceptibility to infection by SARS-CoV2 [5-8]. Observational studies have reported that patients taking ACE-i/ARBs treatment are at increased risk of becoming infected with SARS-CoV2 and developing severe forms of COVID-19 disease.

During the pandemic, contradictory studies and opinions have suggested that local inactivation of the reninangiotensin aldosterone system may have protective effects against the development and progression of acute lung failure [3, 9]. Professional societies have navigated this ambiguity by recommending that patients should continue to use ACE inhibitors or ARBs [10]. However, these recommendations have not been supported by medical data. In consequence, the clinical decision-making process has 
become even more difficult for physicians until this interaction is clarified with robust clinical data. The aim of this study was to assess the behaviors of physicians toward prescribing ACE-i/ARBs treatment during the COVID-19 pandemic to determine if physicians had changed their routine treatment algorithms and how they had been affected by these speculations.

\section{Materials and methods}

This cross-sectional survey included cardiologists, internists, and family physicians responsible for the treatment of hypertension or prescribing antihypertensive drugs. The study was administered in six metropolitan areas located in central and western Turkey, between 1 May and 19 May 2020. The hospitals and family health centers from the mentioned cities were selected according to their COVID19 patient loads. The questionnaire was developed in Turkish by an expert committee including the manuscript authors. Although the surveys were not formally validated, the development process involved comprehensive reviewing and piloting of the surveys. The survey consisted of 26 questions about current hypertension treatment behavior with ACE-i and ARBs during the COVID-19 pandemic. Physicians who were responsible for the treatment of at least 30 patients with hypertension per month were invited to participate in the survey by phone or e-mail. The survey was delivered as an online form and responses were submitted online. The study was approved by the Ethics Committee of Izmir Tepecik Training and Research Hospital (approval number 2020/ 6-11).

The sample size was calculated to be 213 to provide $80 \%$ power with an effect size of 0.5 at an alpha of 0.05 using open source software, Open-epi Version 3.01. A convenience sample of 460 physicians was approached by phone or e-mail at hospital and family health centers in central and western Turkey. A total of 232 physicians completed the survey, presenting a response rate of 50.4\%. Data obtained in the study were analyzed statistically using SPSS for Windows Version 15.0 software (SPSS Inc., Chicago, IL, USA). Descriptive statistics were presented using tables of frequencies for the ordinal variables and median and interquartile range values for the appropriate variables. The primary outcome was the proportion of subjects changing the prescribing pattern of RAAS blockers. The Pearson $\chi 2$ test was used to identify significant relationships between categorical variables. To determine whether physician specialist training contributed to the behaviors of hypertension treatment, the physicians were separated into three groups based on their specialist area (family medicine, Internal medicine, and cardiology). The Kruskal-Wallis test was used to assess differences between specialists in attitudes and behaviors about hypertension treatment and to assess multiple group comparisons for ordinal variables. Bonferroni correction was performed to adjust for multiple group comparisons. A value of $p<0.05$ was considered statistically significant.

\section{Results}

\section{Baseline features}

A total of 232 physicians completed the survey in the defined study period and 220 physicians (142 male, 78 female) gave permission for the data retrieved from the questionnaire to be analyzed within the framework of the study. A total of 12 physicians were excluded from the study as they managed $<30$ patients with hypertension per month. Most of the participants (63.2\%) were aged $<40$ years and $12.8 \%$ of them were $>50$ years of age. Nearly half of the physicians participating in the survey were cardiologists $(n=88,40 \%) 71(32.3 \%)$ were family medicine/ general practitioners and $61(27.7 \%)$ were internal medicine specialists. Of the physicians, $111(50.4 \%)$ were working in tertiary level healthcare centers (University/Training and research hospital). In respect of experience, half of the participants $(53 \%)$ had $>10$ years of experience in practice (Table 1).

\section{Knowledge of interaction between ACE-i/ARBs and COVID-19}

Of the total physicians in the study, $65 \%$ described themselves as having a moderate to high level of knowledge about the relationship between COVID-19 infection and ACE-i/ARBs treatments and $16 \%$ of the study population stated that they did not have enough information on this subject. The sources of information showed variety but $83.9 \%$ of the physicians were informed from at least one reliable academic document such as reports of scientific societies and articles of medical journals. When the respondents were asked to evaluate the reliability of the evidence of these sources, $84 \%$ stated that the reports were suspicious /contradictory and emphasized that new studies are needed. The opinions of the physicians about the relationship between COVID-19 infection and ACE-i/ARBs treatments are shown in Fig. 1a.

\section{Changes in prescribing pattern}

Requests from patients to change ACE-i/ARBs treatment during the pandemic were reported to have been received by $65 \%(143 / 220)$ of the physicians. Of the total physicians, $78.7 \%(173 / 220)$ advised continuing the ACE-i/ARBs 
Table 1 Characteristics of the physicians participating in the survey.

\begin{tabular}{lll}
\hline & $n$ & $\%$ \\
\hline Gender (Male/Female) & $142 / 78$ & $64.5 / 35.5$ \\
Age & & \\
$20-29$ & 39 & 17.7 \\
$30-39$ & 100 & 45.4 \\
$40-49$ & 53 & 24.1 \\
$\quad$ 50 & 28 & 12.8 \\
Specialty & & \\
Family medicine/General practitioner & 71 & 32.3 \\
$\quad$ Internal medicine & 61 & 27.7 \\
Cardiology & 88 & 40 \\
Seniority & & \\
$\quad$ Assistant/general practitioner & 83 & 37.7 \\
Specialist & 97 & 44.1 \\
$\quad$ Assoc. Prof./Prof. & 40 & 18.2 \\
Duration of practice & & \\
1-10 years & 104 & 47.3 \\
11-20 years & 77 & 35 \\
>20 years & 39 & 17.7 \\
Hospital & & \\
Primary care hospital & 56 & 25.5 \\
Secondary care hospital & 53 & 24.1 \\
Tertiary hospital & 111 & 50.4 \\
\hline
\end{tabular}

medications, $12.7 \%$ (28/220) left the decision to the patient, having explained that there were conflicting views on this issue, and 8.6\% (19/220) discontinued the treatment and switched to another drug.

\section{Reliability of ACE-i/ARBs in different conditions}

The respondents were asked to score their level of reliance of ACE-i/ARBs treatments in different clinical conditions on a 10-point Likert scale from 1 to 10. Questions were then asked to ascertain the probability of the physician beginning and repeating ACE-i/ARBs treatment during the COVID-19 pandemic. These items were also scored using a Likert scale. The median ( \pm interquartile range) score indicating the general reliance level of physicians in ACE-i/ARBs therapy was $8 \pm 4$ and for the trend to start new ACE-i/ARBs therapy was $8 \pm 4$. The median score indicating withholding the current ACEi/ARBs therapy was $1 \pm 1$ and for the continuation of the therapy was $9 \pm 1$ (Fig. 2). The tendency for changing the prescribing patterns of ACE-i/ARBs treatment in different clinical conditions, including coronary artery disease, heart failure, hypertension alone, and multiple comorbidities is shown with box-plot graphs in Fig. 3 .

\section{Effect of experience on knowledge level}

Although more than half of the specialists did not believe there was any relationship between ACE-i/ARBs use and risk of having COVID-19 disease, only one-third of the assistant doctors/general practitioners held these beliefs. Nearly $40 \%$ of the assistant doctors believed that ACE-i/ ARBs users had more-severe COVID-19 disease. The effect of experience on knowledge level and on the prescribing pattern was evaluated. There was determined to be a close interaction with every 5 years increase of experience in practice showing a steady increase in the high level of knowledge ratio about ACE/ARB treatment and COVID-19 disease. (Fig. 4).

\section{Effects of physicians' specialties}

The percentage of physicians who thought they had a high level of knowledge about ACE-i/ARB treatment and COVID -19 interaction was $48.7 \%$ in cardiologists, $23.7 \%$ in internal medicine, and $11.5 \%$ in family medicine (Fig. 5a). The percentage of physicians who considered that ACE-i/ARBs therapy may be harmful during the COVID19 pandemic was $44.2 \%$ of the family medicine specialists, $34.8 \%$ of the internal medicine specialists, $33.3 \%$ of the general practitioners, and $15.4 \%$ of the cardiologists. Conversely, $27 \%$ of cardiologists, $13 \%$ of internal medicine specialists, $8 \%$ of general practitioners, and $6 \%$ of family medicine physicians had positive opinions that these medications could be associated with less severe disease and could be protective against infection of COVID-19 disease (Fig. 5b).

More than two-thirds (76.2\%) of the physicians did not change their mind, $27.6 \%$ of cardiologists changed their opinion in favor of ACE-i/ARBs treatment and $16.6 \%$ of general practitioners were affected negatively by the environment of uncertainty during the COVID-19 pandemic. Calcium channel blockers (CCB) were the most preferred agents in the case of ACE-i/ARBs cessation (Fig. 5c). It was observed that $14 \%$ of all physicians, $19 \%$ of cardiologists and $24 \%$ of internal medicine doctors considered changing the ACE-i/ARBs therapy in the case of hypertension and multiple comorbidities respectively. The general reliance level was highest among cardiologists (8.0 $\pm 4)$ and lowest $(5.0 \pm 3)$ among the family medicine/general practitioners $(p<0.05)$. The strategy for repeating therapy was the same in all physicians, and the practice of starting new ACE-i/ARBs reported by the cardiologists was statistically different from that of both internal medicine and family medicine/general practitioners $(8.0 \pm 4$ vs $8.0 \pm 4$ vs $9.0 \pm 3, p<0.05$ ) (Table 2). 
Fig. 1 Answers of the physicians to level of knowledge questions about the relationship between $\mathrm{ACE}$ / ARB treatment and Covid-19. a What types of relationship between COVID-19 infection and ACE/ARB treatments?. b How do you find your level of knowledge about the relationship between COVID-19 infection and ACE /ARB treatments? c Do you think the publications showing the relationship between COVID-19 infection and ACE/ARB are convincing?.

A- What types of relationship between COVID-19 infection and ACE / ARB treatments ?

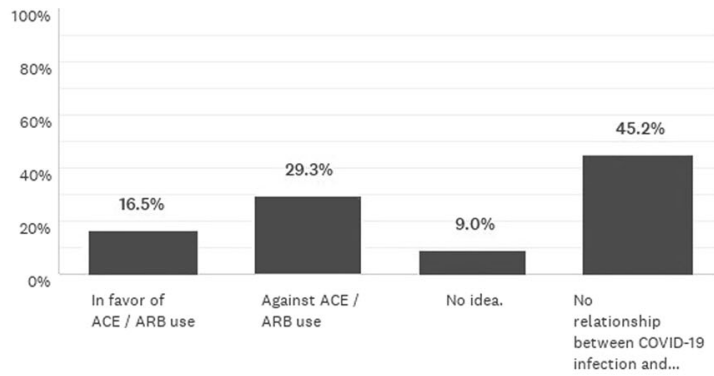

B- How do you find your level of knowledge about the relationship between COVID-19 infection and ACE / ARB treatments?

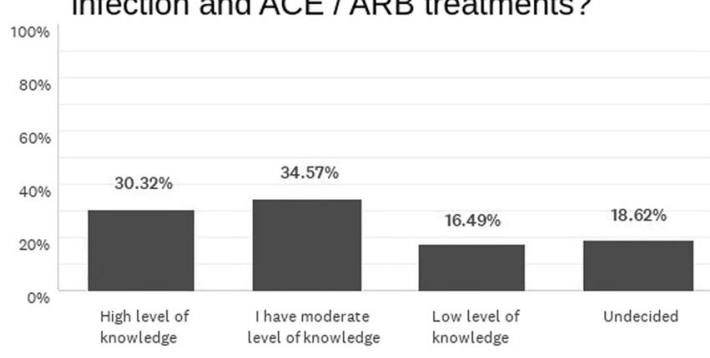

C- Do you think the publications showing the relationship between COVID 19 infection and ACE / ARB are convincing?

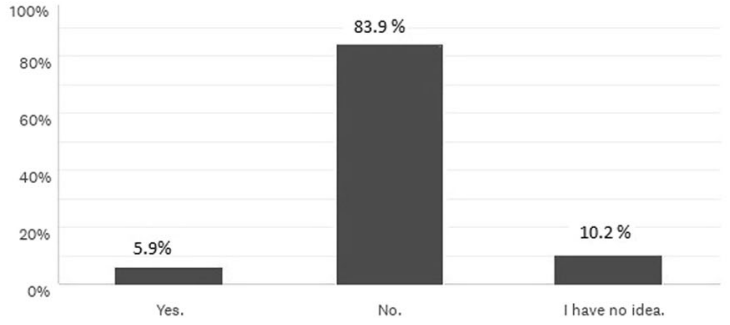



Fig. 2 Box plots showing the level of reliance in ACE-i/ARBs treatments by physicians; general reliance level; reliance level while repeating therapy; reliance level while starting new ACE-i/ARBs; reliance level for cessation the treatment.

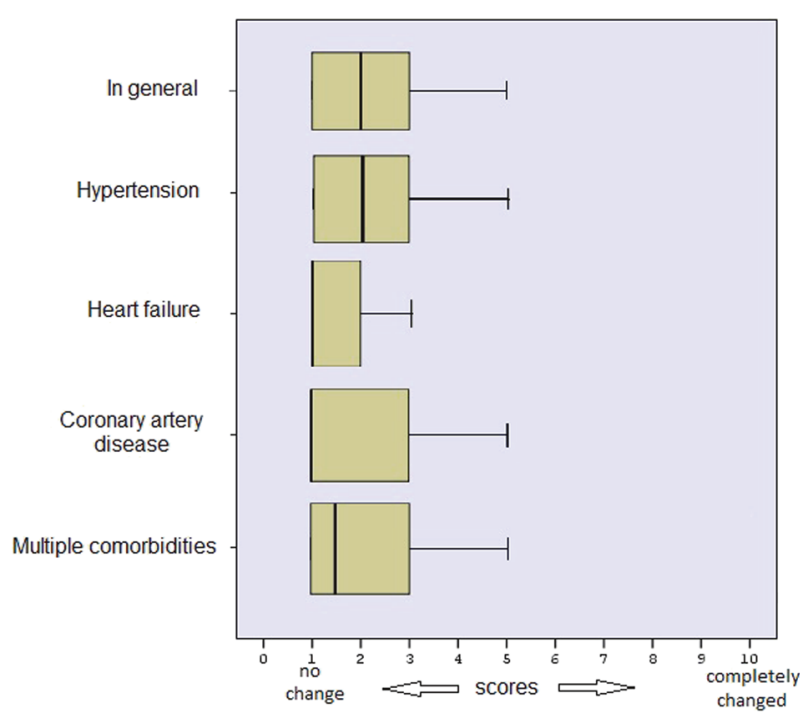

Fig. 3 The tendency for changing the patterns of ACE-i/ARBs treatments by physicians in different clinical conditions; in general, in hypertensive patients; in heart failure patients; in coronary artery disease; in patient with multiple comorbidities. 
Fig. 4 Effect of the experience on level of knowledge of the physicians. a How do you find your level of knowledge about the relationship between COVID-19 infection and ACE /ARB treatments? b What types of relationship between COVID19 infection and ACE/ARB treatments?.
A- How do you find your level of knowledge about the relationship between COVID-19 infection and ACE / ARB treatments?

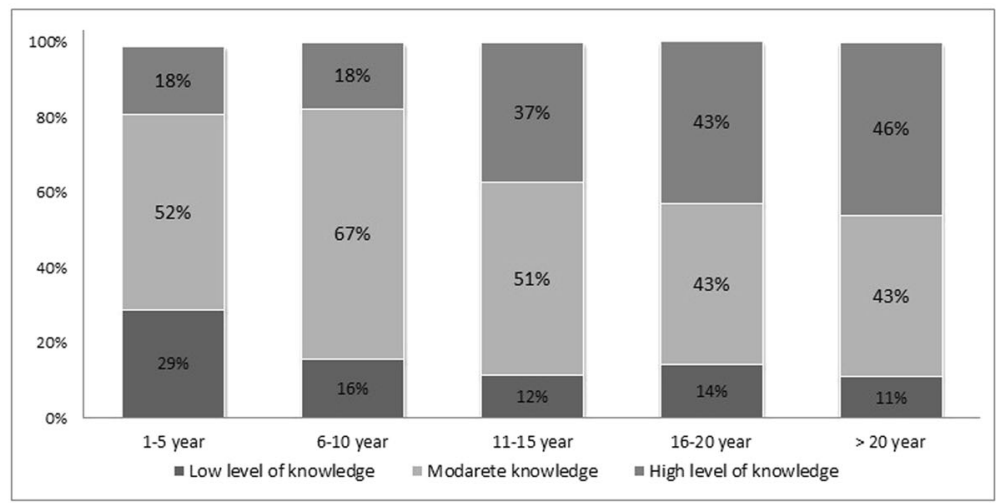

B- What types of relationship do you think between COVID-19 infection and ACE / ARB treatments?

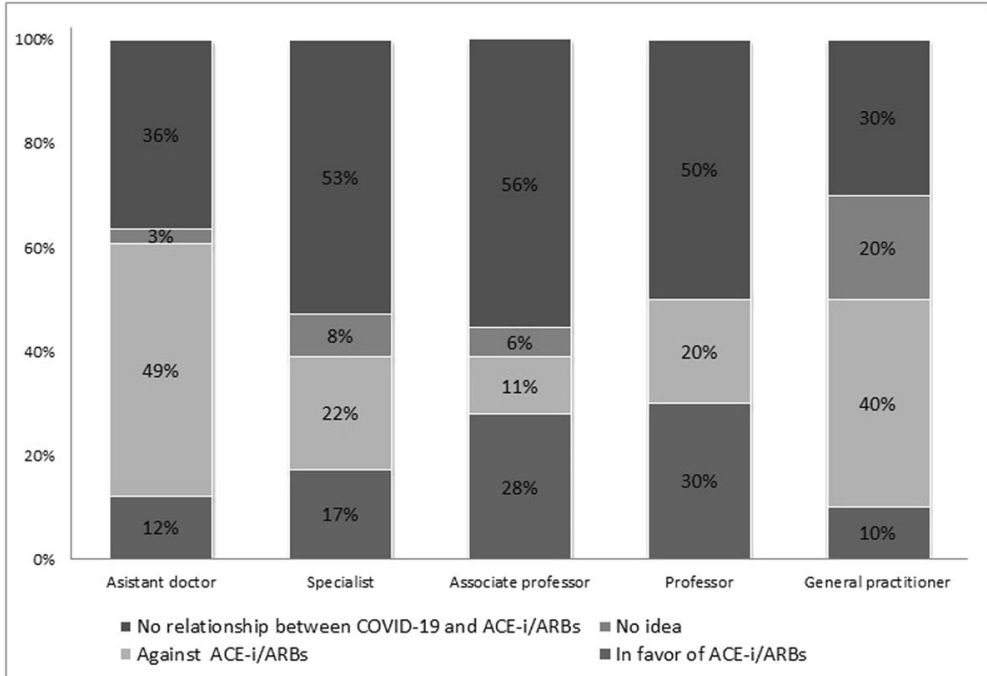

\section{Effect of duration on practice and working hospital}

The institutions where the respondents worked were categorized in three levels as primary, secondary, and tertiary in order to evaluate the effects of the type of healthcare facility on physician's behavior while starting or stopping ACEI/ ARB treatments. The level of the healthcare institution and the duration of working were found to have no impact on repeating/starting/general practice about ACE-i/ARBs (Table 3).

\section{Discussion}

By mid-June 2020, at the time of writing this study, 8.5 million people have been infected with SARS-CoV-2 and over 470,000 have died worldwide. More and more patients are still becoming infected every day. As
COVID-19 disease is caused by the SARS-CoV-2 virus, some speculations have been put forward linking aminopeptidase ACE2 with this disease [11]. Based on these speculations, arguments and concerns about these medications have arisen in the community. Despite the supporting reports of scientific societies against discontinuation of these drugs, the lack of information at the beginning of the pandemic might have had an impact on the behaviors of physicians in respect of whether or not to prescribe [12].

Early reports, which mainly came from China, raised suspicion and encouraged doctors to stop prescribing ACEi/ARBs [13]. These suggestions come from observational studies of large patient populations from different countries [14-16]. In these trials, researchers observed that hypertensive patients had more-severe COVID-19 disease than other patients, so concluded that, hypertension could be a risk factor for a severe disease course [17]. Nevertheless, 
Fig. 5 Effects of physicians'

specialties on level of

knowledge and treatment

preferences. a Effect of

speciality on level of

knowledge. b Types of

relationship according to

speciality. c Effect of speciality

on switching preferences.

\section{A- Effect of speciality on level of knowledge}

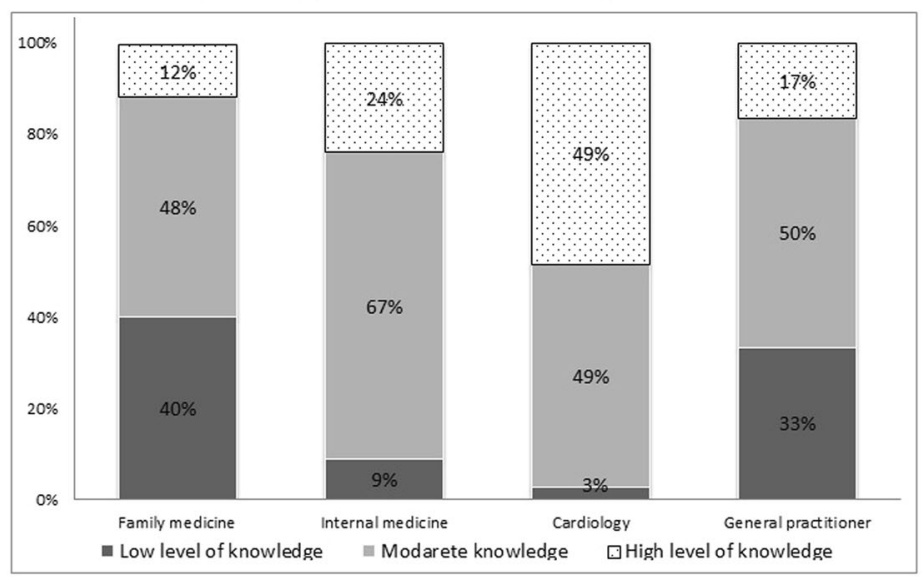

B- Types of relationship according to speciality

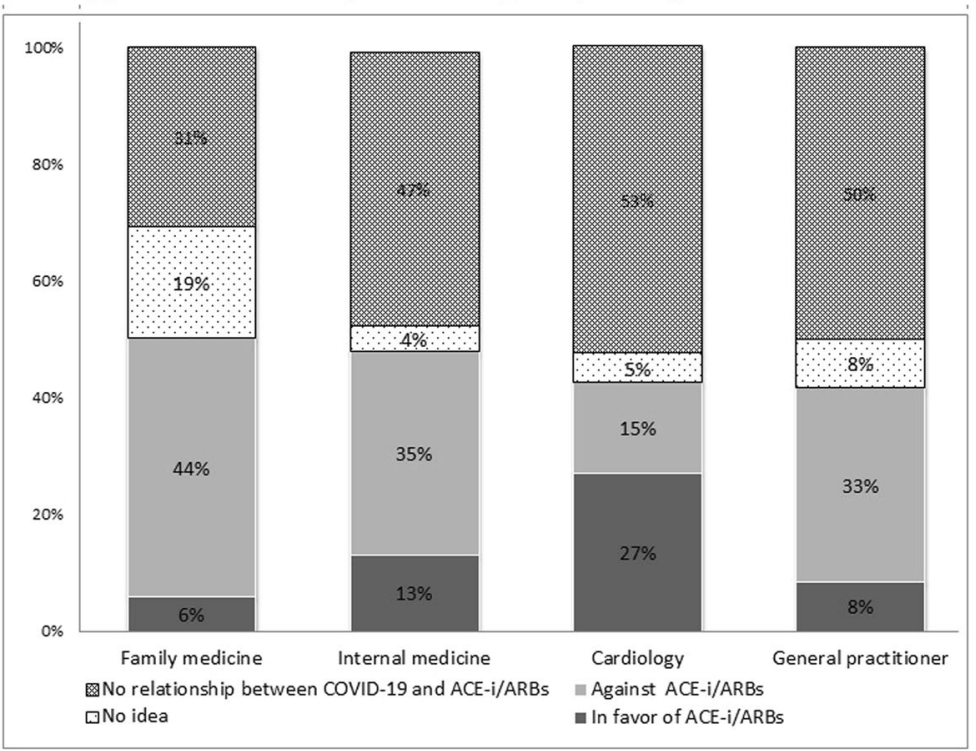

\section{C- Effect of speciality on switching preference}

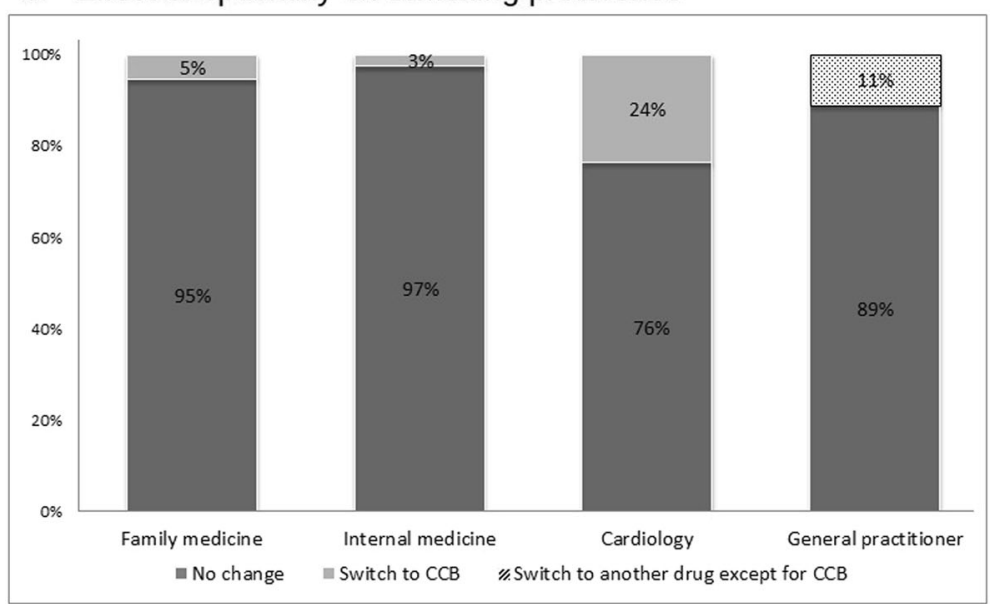

the hypertensive patients with severe COVID-19 in those studies had poorer general health as they had additional risk factors such as older age and comorbidities (heart failure, diabetes, chronic kidney disease).These preliminary results not taking these potential confounding factors into account may have caused cessation of these important beneficial and 
Table 2 Effect of specialty on reliance level of ACE-i/ARBs and changing behavior of prescribing pattern in different clinical conditions.

\begin{tabular}{|c|c|c|c|c|}
\hline Speciality & $\begin{array}{l}\text { Family medicine/general } \\
\text { practitioner }\end{array}$ & Internal medicine & Cardiologist & $p$ value \\
\hline \multicolumn{5}{|c|}{ Reliance level of ACE-i/ARBs } \\
\hline General reliance & $5.0 \pm 3$ & $8.0 \pm 4$ & $8.0 \pm 4$ & $0.02^{\mathrm{a}}$ \\
\hline Repeating ACE/ARB & $9.0 \pm 5$ & $9.0 \pm 2$ & $9.0 \pm 6$ & 0.31 \\
\hline Starting New ACE/ARB & $8.0 \pm 4$ & $8.0 \pm 4$ & $9.0 \pm 3$ & $0.009^{a, b}$ \\
\hline \multicolumn{5}{|c|}{ Changing prescribing pattern in different clinical conditions } \\
\hline General & $2.0 \pm 4$ & $2.0 \pm 2$ & $1.0 \pm 2$ & 0.09 \\
\hline Hypertension & $1.0 \pm 3$ & $2.0 \pm 2$ & $2.0 \pm 3$ & 0.80 \\
\hline Heart failure & $1.5 \pm 3$ & $1.0 \pm 2$ & $1.0 \pm 0$ & $0.005^{\mathrm{a}, \mathrm{b}}$ \\
\hline Coronary artery disease & $1.5 \pm 2$ & $1.0 \pm 1$ & $1.0 \pm 1$ & 0.25 \\
\hline Multiple comorbidities & $2.0 \pm 4$ & $2.0 \pm 2$ & $1.0 \pm 2$ & 0.18 \\
\hline
\end{tabular}

The data are expressed as median \pm interquartile range.

a'Between Family Medicine/General practitioner and Cardiologist $p<0.05$.

${ }^{\mathrm{b}}$ Between Internal medicine and Cardiologist $p<0.05$.
Table 3 Effect of duration on practice and working center on reliance level of ACE-i/ARBs.

\begin{tabular}{llll}
\hline $\begin{array}{l}\text { Reliance level of ACE-I / } \\
\text { ARBs }\end{array}$ & $\begin{array}{l}\text { General } \\
\text { reliance }\end{array}$ & $\begin{array}{l}\text { Repeating } \\
\text { ACE-I } / \\
\text { ARBs }\end{array}$ & $\begin{array}{l}\text { Starting new } \\
\text { ACE-i/ } \\
\text { ARBs }\end{array}$ \\
\hline $\begin{array}{l}\text { Duration on practice (years) } \\
1-10\end{array}$ & $8.0 \pm 3$ & $9.0 \pm 2$ & $8.0 \pm 4$ \\
$11-20$ & $8.0 \pm 4$ & $9.0 \pm 5$ & $9.0 \pm 4$ \\
$>20$ & $7.5 \pm 4$ & $9.5 \pm 5$ & $8.5 \pm 3$ \\
$p$ value (Between groups) & 0.89 & 0.11 & 0.27 \\
Working hospital & & & \\
$\quad$ Primary Health center & $7.0 \pm 3$ & $10.0 \pm 1$ & $8.0 \pm 4$ \\
$\quad$ Secondary health center & $8.0 \pm 4$ & $9.0 \pm 1$ & $9.0 \pm 5$ \\
$\quad$ Tertiary center & $8.0 \pm 3$ & $9.0 \pm 2$ & $8.0 \pm 5$ \\
$\quad p$ value (Between groups) & 0.66 & 0.10 & 0.09 \\
\hline
\end{tabular}

The data are expressed as median \pm interquartile range.

lifesaving medications in the absence of supporting evidences.

More recently, at later stages of the pandemic, more sophisticated studies were published, which mainly aimed to clarify whether ACE-i/ARBs were harmful and to propose risk factors for severe COVID-19 disease with multivariate logistic-regression analysis. However, some researchers have argued that ACE-i/ARBs may have some protective effects against COVID-19 disease. It has been suggested that ACE2 upregulation by ACE-i/ARBs inhibitors may decrease unopposed angiotensin II activity, which contributes to facilitating neutrophil infiltration and causes organ injury in response to bacterial endotoxin $[18,19]$. In a small study from China, Liu et al. found that elevated levels of plasma angiotensin II were correlated with viral load and degree of lung injury [20]. Prior to that study, there had been studies of other viruses showing that recombinant ACE2 administration reversed lung injury [21, 22]. In the current study, $16.7 \%$ of the respondents believed that ACEi/ARBs treatment was associated with less severe disease or a low likelihood of being infected. A recent study conducted in Italy clearly showed that neither ACE inhibitors nor ARBs were harmful during the COVID-19 outbreak [23].

In the current study, while physicians expressed many concerns regarding ACEi/ARBs, nearly half believed that ACE-i/ARBs treatment may worsen the clinical scenario and increase the probability of getting infected. However, two-thirds of the physicians had not changed their antihypertensive medication prescribing pattern. The results suggest that the physician's behavior toward hypertension treatment had not changed easily based on speculations or concerns during the pandemic in Turkey.

With the argument regarding hypertension as a risk factor for COVID- 19, studies have been conducted to determine which antihypertensive medications are the safest. Reynolds et al. assessed the relationship between five common classes of antihypertensive medications (ACE-i/ ARBs, beta-blockers, calcium-channel blockers, thiazide diuretics) and COVID-19 [24]. There was reported to be no association for any of the mentioned drug classes. In the current study, majority of respondents stated that they continued as before and had made no change in their treatment regimen, and only a small number of participants emitted that they were hesitant to start new ACE-i/ARBs therapies. Ca channel blockers were considered a safe alternative in cases where ACE-i/ARBs drugs were discontinued or changed, and $12.9 \%$ of physicians reported that they preferred CCB instead of ACE-i/ARBs. 
From the results of this study, it appears that practicing physicians found less risk in changing their prescribing behavior in cases with hypertension alone. Nevertheless, they considered continuing with RAAS inhibitors, which are cornerstone therapies in heart failure patients. SARS$\mathrm{CoV}-2$ virus is a cardiotropic virus than can cause severe myocarditis in up to $40 \%$ of critically ill patients [25]. Cessation of ACE-i/ARBs treatment may lead to decompensation in those with previous heart failure and results in SARS-CoV-2 infected patients being more susceptible to myocardial damage.

An interesting new hypothetical study from England investigated the connection of influenza infection with SARS disease via the shared mechanism of both for entering the human cells by ACE2 receptors [26]. The use of ACE-i/ARBs therapies was found to be associated with either no effect on the incidence of influenza or possibly a much lower incidence, depending on the duration of use. Based on this trial, it was suggested that the same relationship can be seen in SARS-CoV-2.

This study had several limitations. The overall sample size was small and the subgroups were not normalized to the practicing population of family medicine, internal medicine, cardiologist, and general practitioner. In addition, the questionnaire that was used had not been previously validated. In the literature, there are no other studies that have utilized a questionnaire to measure physicians' attitudes regarding SARS-CoV-2 and hypertension treatment interactions. The study population was sampled at hospitals and family health centers in six major cities in Turkey, so the results might not be reflective of physicians practicing in the whole of Turkey. The physicians interested in this issue might have agreed to participate in the survey, which could have led to the high rate of physicians who did not change the treatment strategy.

\section{Conclusion}

The present survey is the first study to have provided a snapshot showing behaviors of Turkish physicians toward prescribing ACE-i/ARBs treatment during the COVID-19 pandemic. An important finding of this study is that the majority of physicians were not affected by the concerns, which had arisen at the beginning of the pandemic and continued to prescribe ACE-i/ARBs treatments. However, there was a statistically significant difference between different physician specialties in the reliance level in different clinical situations. Given the probability of emerging new evidence on this matter, additional studies should be conducted to clarify the changes in prescribing habits of physicians of antihypertensive medications during the course of the COVID-19 pandemic.

\section{Summary}

\section{What is known about topic}

- In the first half of 2020 COVID-19 infection became pandemic. SARS-CoV-2 virus enters into cells by binding to the ACE2 receptor.

- Hypertension is the most common comorbity in COVID-19 patient. In early studies, it was speculated that ACE/ARB users may be more susceptible to COVID-19 disease and have worse outcomes.

- Concerns about ACE/ARB use have arisen among healthcare professionals. Scientific societies against discontinuation of these drugs.

\section{What this study adds}

- In pandemic era, general reliance level of in ACE-i/ ARBs therapy was high. $78.7 \%$ of the physicians advised continuing the ACE-i/ARBs medications.

- Calcium channel blockers were the most preferred agents in the case of ACE-i/ARBs cessation due to safety concerns.

- The strategy for repeating therapy was the same in all specialities. The practice of starting new ACE-i/ARBs and reliance level in different clinical situations showed significant difference between physician specialties.

Funding This research received no specific grant from any funding agency in the public, commercial or not-for-profit sectors.

\section{Compliance with ethical standards}

Conflict of interest The authors declared no potential conflicts of interest with respect to the research, authorship and/or publication of this article.

Informed consent All subjects gave informed consent for participation.

Publisher's note Springer Nature remains neutral with regard to jurisdictional claims in published maps and institutional affiliations.

\section{References}

1. Li XC, Zhang J, Zhuo JL. The vasoprotective axes of the reninangiotensin system: physiological relevance and therapeutic implications in cardiovascular, hypertensive and kidney diseases. Pharm Res. 2017;125:21-38.

2. Zheng Y-Y, Ma Y-T, Zhang J-Y, Xie X. COVID-19 and the cardiovascular system. Nat Rev Cardiol. 2020;17:259-60.

3. Vaduganathan M, Vardeny O, Michel T, McMurray JJV, Pfeffer MA, Solomon SD. Renin-Angiotensin-Aldosterone System Inhibitors in Patients with Covid-19. N Engl J Med. 2020;382:1653-9. 
4. Li W, Moore MJ, Vasilieva N, Sui J, Wong SK, Berne MA, et al. Angiotensin-converting enzyme 2 is a functional receptor for the SARS coronavirus. Nature. 2003;426:450-4.

5. Ocaranza María Paz, Godoy Ivan, Jalil Jorge E, Varas Manuel, Collantes Patricia, Pinto Melissa, et al. Enalapril Attenuates Downregulation of Angiotensin-Converting Enzyme 2 in the Late Phase of Ventricular Dysfunction in Myocardial Infarcted Rat. Hypertension 2006;48:572-8.

6. Huang M, Li X, Meng Y, Xiao B, Ma Q, Ying S, et al. Upregulation of angiotensin-converting enzyme (ACE) 2 in hepatic fibrosis by ACE inhibitors. Clin Exp Pharm Physiol. 2010;37: e1-6.

7. Yuichiro Ishiyama, Gallagher Patricia E, Averill David B, Ann TallantE, Bridget BrosnihanK, Ferrario Carlos M. Upregulation of Angiotensin-Converting Enzyme 2 After Myocardial Infarction by Blockade of Angiotensin II Receptors. Hypertension. 2004;43:970-6.

8. Ferrario Carlos M, Jewell Jessup, Chappell Mark C, Averill David B, Bridget BrosnihanK, Ann TallantE, et al. Effect of Angiotensin-Converting Enzyme Inhibition and Angiotensin II Receptor Blockers on Cardiac Angiotensin-Converting Enzyme 2. Circulation $2005 ; 111: 2605-10$.

9. Fernández-Ruiz I. RAAS inhibitors do not increase the risk of COVID-19. Nat Rev Cardiol. 2020;17:383-383.

10. Nadar SK, Tayebjee MH, Stowasser M, Byrd JB. Managing hypertension during the COVID-19 pandemic. J Hum Hypertens. 2020;34:415-7.

11. Doumas M, Patoulias D, Katsimardou A, Stavropoulos K, Imprialos K, Karagiannis A. COVID19 and increased mortality in African Americans: socioeconomic differences or does the renin angiotensin system also contribute? J Hum Hypertens. 2020;1-4. https://doi.org/10.1038/s41371-020-0380-y. Epub ahead of print.

12. Bozkurt B, Kovacs R, Harrington B. Joint HFSA/ACC/AHA Statement Addresses Concerns Re: using RAAS Antagonists in COVID-19. J Card Fail. 2020;26:370.

13. Fang L, Karakiulakis G, Roth M. Are patients with hypertension and diabetes mellitus at increased risk for COVID-19 infection? Lancet. Respir Med. 2020;8:e21.

14. Guan W-J, Ni Z-Y, Hu Y, Liang W-H, Ou C-Q, He J-X, et al. Clinical Characteristics of Coronavirus Disease 2019 in China. N Engl J Med. 2020;382:1708-20.
15. Huang C, Wang Y, Li X, Ren L, Zhao J, Hu Y, et al. Clinical features of patients infected with 2019 novel coronavirus in Wuhan, China. Lancet 2020;395:497-506.

16. Grasselli G, Zangrillo A, Zanella A, Antonelli M, Cabrini L, Castelli A, et al. Baseline Characteristics and Outcomes of 1591 Patients Infected With SARS-CoV-2 Admitted to ICUs of the Lombardy Region, Italy. JAMA. 2020;323:1574-81.

17. Huang S, Wang J, Liu F, Liu J, Cao G, Yang C, et al. COVID-19 patients with hypertension have more severe disease: a multicenter retrospective observational study. Hypertension Res. 2020;43:824-31.

18. Gurwitz D. Angiotensin receptor blockers as tentative SARSCoV-2 therapeutics. Drug Dev Res. 2020;81:537-40.

19. Sodhi CP, Wohlford-Lenane C, Yamaguchi Y, Prindle T, Fulton WB, Wang S, et al. Attenuation of pulmonary ACE2 activity impairs inactivation of des-Arg9 bradykinin/BKB1R axis and facilitates LPS-induced neutrophil infiltration. Am J Physiol Lung Cell Mol Physiol. 2018;314:L17-31.

20. Liu Y, Yang Y, Zhang C, Huang F, Wang F, Yuan J, et al. Clinical and biochemical indexes from 2019-nCoV infected patients linked to viral loads and lung injury. Sci China Life Sci. 2020;63:364-74.

21. Gu H, Xie Z, Li T, Zhang S, Lai C, Zhu P, et al. Angiotensinconverting enzyme 2 inhibits lung injury induced by respiratory syncytial virus. Sci Rep. 2016;6:19840.

22. Zou Z, Yan Y, Shu Y, Gao R, Sun Y, Li X, et al. Angiotensinconverting enzyme 2 protects from lethal avian influenza A H5N1 infections. Nat Commun. 2014;5:3594.

23. Mancia G, Rea F, Ludergnani M, Apolone G, Corrao G. Renin-Angiotensin-Aldosterone System Blockers and the Risk of Covid-19. N Engl J Med. 2020;382:2431-40.

24. Reynolds HR, Adhikari S, Pulgarin C, Troxel AB, Iturrate E, Johnson SB, et al. Renin-Angiotensin-Aldosterone System Inhibitors and Risk of Covid-19. N Engl J Med. 2020;382:2441-8.

25. Arentz M, Yim E, Klaff L, Lokhandwala S, Riedo FX, Chong M, et al. Characteristics and Outcomes of 21 Critically Ill Patients With COVID-19 in Washington State. JAMA. 2020;323:1612-4.

26. Chung S-C, Providencia R, Sofat R. Association between Angiotensin Blockade and Incidence of Influenza in the United Kingdom. N Engl J Med. 2020;383:397-400. 